\title{
Erratum: Control of grain size and rice yield by GL2-mediated brassinosteroid responses
}

Ronghui Che, Hongning Tong, Bihong Shi, Yuqin Liu, Shanru Fang, Dapu Liu, Yunhua Xiao, Bin Hu, Linchuan Liu, Hongru Wang, Mingfu Zhao and Chengcai Chu

Nature Plants 1, 15195 (2015); published online 21 December 2015; corrected 13 January 2016

In the version of this Letter originally published, several instances of GL2 should have been italic to represent the gene rather than the protein. This has now been corrected. 